Interview

\title{
Aus der Praxis für die Praxis: der Radiologiekongress Nord 2018
}

Von der Oberärztin bis zum Medizinstudenten: Die gemeinsame Jahrestagung der Norddeutschen Röntgengesellschaft und der Röntgengesellschaft von Niedersachsen, Bremen und Sachsen-Anhalt bietet ein abwechslungsreiches und praxisbezogenes Fortbildungsprogramm. Welche Highlights erwarten die Teilnehmerinnen und Teilnehmer am 16. und 17. Februar 2018 in Hamburg? Im Interview gibt PD Dr. Roger Eibel aus Schwerin, Kongresspräsident des Radiologiekongresses Nord 2018, erste Einblicke.

Herr Dr. Eibel, der Norddeutsche Röntgenkongress 2018 nimmt die Themenschwerpunkte Muskulo-Skelettal, Interventionen, Abdomen, Mamma und Urogenital in den

\section{Blick. Warum haben Sie diesen Fokus gewählt?}

2018 möchte ich fortsetzen, wofür der Radiologiekongress Nord bereits in den letzten Jahren stand: ein Fortbildungskongress zu sein. Ich habe Themen ausgewählt, die wirklich relevant sind, die den Berufsalltag prägen und zu denen sich jeder von uns wünscht, noch etwas mehr zu wissen: also aus der Praxis für die Praxis. Daher ist mir wichtig, dass in den Vorträgen die Bedeutung für unsere tägliche Arbeit dominiert. Natürlich immer auf der Basis von wissenschaftlich gesicherten Erkenntnissen. Dabei versteht sich von selbst, dass wir in den zwei Tagen nicht das Gesamtgebiet der Radiologie abdecken können. Unter Beach-

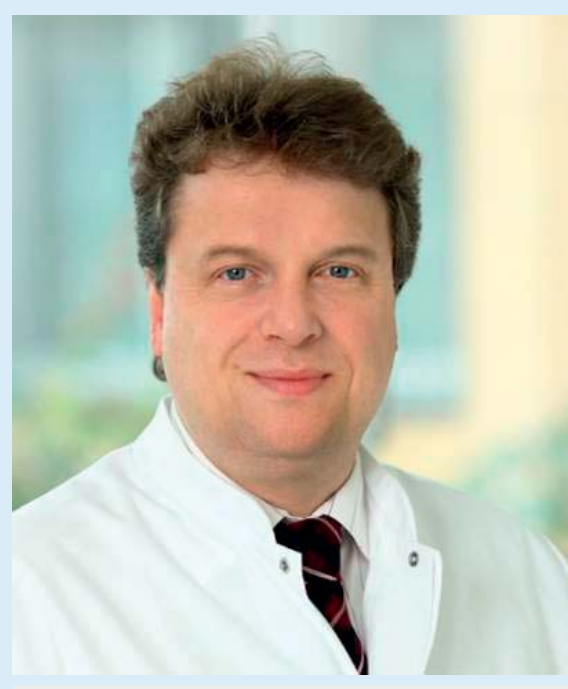

PD Dr. Roger Eibel 


\section{RADIOLOGIEKONGRESSNORD2018}

\section{DER FORTBILDUNGSKONGRESS IM NORDEN}

tung der Kongressprogramme der letzten Jahre, dem Anforderungsprofil an Radiologen in Klinik und Praxis und aus den jüngsten Entwicklungen ergaben sich dann die Themenschwerpunkte Muskulo-Skelettal, Mamma, Urogenital und Abdomen. Und natürlich ist die Radiologie nicht nur Diagnostik, sondern auch Intervention. Auch da müssen wir unser Wissen und unsere Kompetenzen erhalten und weiter ausbauen.

\section{Welche Highlights erwarten die Teilneh- merinnen und Teilnehmer?}

Das sind zum einen die bereits oben genannten Themenschwerpunkte. Besonders im Bereich Muskulo-Skelettal sind wir sehr breit aufgestellt und möchten den Kongressteilnehmern viel notwendiges Wissen für die tägliche Befundung an die Hand geben. Ein weiteres Highlight erwartet die Teilnehmer gleich nach der Eröffnungsveranstaltung mit einer berufspolitischen Panelrunde. Ich habe vier Radiologen als Vertreter einer Uni-Klinik, eines privaten Krankenhauskonzerns, einer standortübergreifenden Groß- und einer Einzelstandortpraxis gebeten, aus ihrem Berufsalltag zu berichten. Welche Entwicklungen, welche Chancen und welche Risiken sehen sie jeweils aus ihrem Blickwinkel? Danach können auch die Kongressteilnehmer Fragen stellen und wir kommen so hoffentlich miteinander ins Gespräch. Und natürlich werden wir auch 2018 wieder eine Person ehren, die sich um die Radiologie im Rahmen der NDRG und darüber hinaus über Jahre hinweg verdient gemacht hat. Mehr sei hier noch nicht verraten. Ebenso verleihen wir wieder den Horst-Seils-Preis für eine exzellente wissenschaftliche Arbeit an eine Kollegin oder einen Kollegen, die oder der als Mitglied der NDRG arbeitet und forscht.
Es fällt auf, dass der Radiologiekongress Nord auch 2018 wieder viel Wissenswertes für sehr unterschiedliche Zielgruppen bereithält. Wen wollen Sie denn mit den Veranstaltungen ansprechen?

Ganz einfach: Jeden, der sich mit Radiologie von Berufswegen beschäftigt. Sowohl Chef-, Ober- und Fachärzte, die seit Jahren etabliert sind, als auch Ärztinnen und Ärzte in Weiterbildung im ersten Jahr, werden passende Vorträge und Workshops finden. Auch Medizinstudenten, die vielleicht die Radiologie für sich erst entdecken möchten, sind herzlich willkommen. Workshops über Uroradiologie und der große Q1-Q2-Herz-CT-Kurs runden das Programm ab und machen es für Kollegen interessant, die noch tiefer in diese Themen einsteigen wollen. Radiologie funktioniert nur, wenn verschiedene Berufsgruppen eng und gut kooperieren. Da für uns als Radiologen die Zusammenarbeit mit den MTRA extrem wichtig ist, ist es auch eine Selbstverständlichkeit, mit diesen Kolleginnen und Kollegen einen gemeinsamen Kongress zu gestalten. Da natürlich von einer guten MTRA bzw. einem guten MTRA andere Fähigkeiten und anderes Fachwissen verlangt werden als von Radiologen, haben wir spezielle Vorträge und Workshops für MTRA im Angebot.

\section{Erstmals wird es beim Radiologiekongress Nord auch ein MTRA-Schülerprogramm geben. Was erwartet den MTRA-Nach- wuchs denn dort?}

Wir möchten uns in diesem Jahr noch stärker den kommenden Generationen öffnen. Warum soll ein Kongress nur für bereits beruflich Etablierte interessant sein? Um den beruflichen Nachwuchs müssen wir uns kümmern. Die Zeiten, wo sich Bewerbungen von MTRA und Ärzten gestapelt haben, sind längst vorbei. Also zeigen wir doch, wie spannend Radiologie sein kann! Sowohl für junge Ärzte, gerade aber auch für MTRASchülerinnen und Schüler. Auf unserem Kon- gress werden versierte MTRA, sozusagen die „alten Hasen“, den Schülern Einstelltechniken zeigen, aber auch Grundlagen von CT und MRT. Und nicht zuletzt geht es um das Thema „Prüfungsängste meistern“ - übrigens nicht nur ein Thema für MTRA-Schüler.

Was macht den Radiologiekongress Nord so besonders für Sie - und gibt es einen Programmpunkt, auf den Sie sich persönlich am meisten freuen?

Wir werden zu allen Themen hochrangige Experten auf dem Gebiet der diagnostischen und interventionellen Radiologie haben. Wenn ich mir die vielen Veranstaltungen so anschaue, möchte ich deshalb gar keinen Programmpunkt gesondert hervorheben. Ich bin dankbar, dass so viele Referenten zugesagt haben und ihr Wissen und ihre Erfahrungen auf dem Kongress weitergeben. Darüber hinaus nehmen wir uns in einer eigenen Session auch Zeit, um Antworten auf die Frage zu finden, was passiert, wenn es zu einem Diagnoseirrtum oder einem Diagnosefehler kommt. Auch da haben wir einen ausgewiesenen Experten zu Gast, auf den ich mich, wie auf alle anderen Vortragenden auch, sehr freue. Ich bin mir sicher, dass wir wirklich gute Themen besetzen und hervorragende Referenten zu Gast haben - da macht die Organisation wirklich Spaß.

\section{Infokasten \\ Radiologiekongress Nord 2018}

16. und 17. Februar 2018 - Hamburg

Radisson Blu Hotel Dammtor

www.roeko-nord.de

Kongresspräsident:

PD Dr. med. habil. Roger Eibel - Helios

Kliniken Schwerin

MTRA-Programm (VMTB):

Marcel Apel - Schön Klinik, HamburgEilbeck 